OESOPHAGUS

\title{
Aerophagia, gastric, and supragastric belching: a study using intraluminal electrical impedance monitoring
}

\author{
A J Bredenoord, B L A M Weusten, D Sifrim, R Timmer, A J P M Smout
}

Gut 2004;53:1561-1565. doi: 10.1136/gut.2004.042945

See end of article for authors' affiliations

ath' affiliations

Correspondence to: Dr A J Bredenoord, Department of

Gastroenterology,

St. Antonius Hospital

PO Box 2500, 3430 EM

Nieuwegein, the

Netherlands;

a.bredenoord@

antonius.net

Accepted for publication 27 April 2004
Background: Patients with aerophagia are believed to have excessive belches due to air swallowing. Intraluminal impedance monitoring has made it possible to investigate the validity of this concept.

Methods: The authors measured oesophageal pH and electrical impedance before and after a meal in 14 patients with excessive belching and 14 healthy controls and identified patterns of air transport through the oesophagus. The size of the gastric air bubble was measured radiographically. In four patients prolonged oesophageal manometry was performed simultaneously.

Results: In all subjects, impedance tracings showed that a significant amount of air is propulsed in front of about a third of the swallow induced peristaltic waves. Two types of retrograde gas flow through the oesophagus (belch) were observed. In the first type air flowed from the stomach through the oesophagus in oral direction ("gastric belch"). In the second type air entered the oesophagus rapidly from proximal and was expulsed almost immediately in oral direction ("supragastric belch"). The incidence of air-containing swallows and gastric belches was similar in patients and controls but supragastric belches occurred exclusively in patients. There was no evidence of lower oesophageal sphincter relaxation during supragastric belches. Gastric air bubble size was not different between the two groups.

Conclusions: In patients with excessive belching the incidence of gaseous reflux from stomach to oesophagus is similar to that in healthy subjects. Their excess belching activity follows a distinct pattern, characterised by rapid antegrade and retrograde flow of air in the oesophagus that does not reach the stomach.
A ir swallowing during eating and drinking is a normal physiological event: in a study in healthy subjects, swallowing a $10 \mathrm{ml}$ liquid bolus was found to be accompanied by ingestion of $8-32 \mathrm{ml}$ of air. ${ }^{1}$ In the upright position ingested air will accumulate in the proximal stomach. It has been shown that distention of the proximal stomach elicits transient relaxations of the lower oesophageal sphincter (LOS) allowing the ingested air to be vented. ${ }^{2-6}$ Ingestion of drinks containing carbon dioxide provokes belching through the same mechanism..$^{78}$ Once the air has passed the LOS and entered the oesophageal body, oesophageal distention leads to upper oesophageal sphincter relaxation, enabling gas to escape to the pharynx. ${ }^{9} 10$

Postprandial belching is normal, with three to four belches per hour occurring with a normal diet. Belching is a common symptom in patients with gastro-oesophageal reflux disease and functional dyspepsia ${ }^{11}$ but can also occur as an isolated symptom. In many patients with troublesome repetitive belching the eructation appears to be the result of a behavioural disorder in which excessive air swallowing is the primary event. This poorly defined disease entity is referred to as aerophagia. Until recently, technical limitations stood in the way of an adequate analysis of the events associated with belching and aerophagia. With the advent of the intraluminal impedance recording technique, it has become possible to monitor the passage of air through the oesophagus, either in aboral or oral direction. ${ }^{12} 13$ The aim of this study was to investigate whether patients with frequent belching indeed swallow excessive amounts of air which leads to gastric distention and gaseous gastro-oesophago-pharyngeal reflux.

\section{METHODS}

\section{Subjects}

We studied 14 healthy volunteers (eight males and six females; mean age 32.8 years, range 23-46 years) and 14 patients (eight males and six females; mean age 57.7 years, range $28-75$ years) with complaints of excessive belching. Extensive diagnostic testing in the patients revealed no organic abnormalities. Written informed consent was obtained from all subjects and the protocol was approved by the medical ethics committees of the University Medical Center Utrecht and the Catholic University of Leuven.

\section{Study protocol}

After an overnight fast, radiographs of the upper abdomen were made in anteroposterior and lateral orientation while subjects were standing. Thereafter a routine oesophageal manometry was performed to determine the distance from nostrils to the LOS. After the manometry, the impedance catheter and the $\mathrm{pH}$ catheter were introduced transnasally and positioned based on the manometric findings (see below). In four of the 14 patients with excessive belching we also introduced a manometric catheter and this catheter recorded pressures for the duration of the study. Subjects were in an upright position and after an adaptation period of at least 10 minutes, recording was started and subjects were asked to minimise head movements and to breathe normally. Patients were asked to press the event marker button on the datalogger every time they had an audible belch. After 45 minutes of continuous recording a standardised meal was consumed consisting of a hamburger (McDonald's Quarter Pounder), 20 grams of fresh onions, 44 grams of chips, and $475 \mathrm{ml}$ of orange juice (in total $967 \mathrm{kCal}$ ). The meal had to be finished in 30 minutes. Postprandially, radiographic images were taken in anteroposterior and lateral direction with the subject in upright position. A postprandial recording period of 90 minutes completed the protocol.

Abbreviations: LOS, lower oesophageal sphincter. 


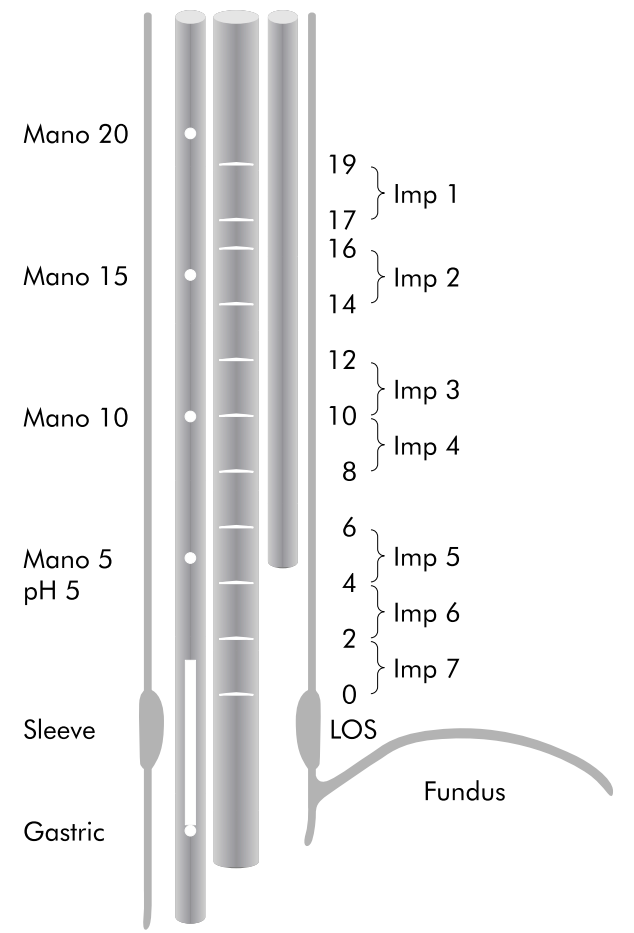

Figure 1 Schematic representation of $\mathrm{pH}$, manometric, and impedancometric assemblies used in this study. From left to right: manometric, impedance, and $\mathrm{pH}$ catheter.

\section{Impedance and $\mathrm{pH}$ monitoring}

For impedance monitoring a seven channel impedance catheter was used (Aachen University of Technology, FEMU, Aachen, Germany). This catheter (outer diameter $2.3 \mathrm{~mm}$ ) enabled recording from seven segments, each recording segment being $2 \mathrm{~cm}$ long. The recording segments were located at $0-2,2-4,4-6,8-10,10-12,14-16$, and $17-19 \mathrm{~cm}$ above the upper border of the manometrically located LOS (fig 1). Impedance signals were stored in a digital system (Aachen University of Technology, FEMU, Aachen, Germany) with a sampling frequency of $1000 \mathrm{~Hz}$. Intraluminal $\mathrm{pH}$ monitoring was performed with a catheter with a glass pH electrode (Ingold AG, Urdorf, Switzerland) and data were stored in a digital datalogger (Orion, MMS, Enschede, the Netherlands) using a sampling frequency of $1 \mathrm{~Hz}$. The $\mathrm{pH}$ glass catheter was positioned $5 \mathrm{~cm}$ above the manometrical upper border of the LOS.

\section{Manometry}

An 18 channel water perfused silicone rubber catheter (outer diameter $4.0 \mathrm{~mm}$, length $75 \mathrm{~cm}$, channel diameter $0.4 \mathrm{~mm}$ ) was used for manometry. Pressures were recorded from four pharyngeal sideholes, four oesophageal sideholes, a sleeve sensor, and a gastric sidehole. This manometric assembly was positioned in such a way that the sleeve sensor membrane straddled the entire LOS (fig 1). All channels were perfused with degassed water at a rate of $0.08 \mathrm{ml} / \mathrm{min}$ using a pneumohydraulic perfusion system (Dentsleeve Pty Ltd, Wayville, South Australia).

Pressures were recorded with external pressure transducers (Abbott, Sligo, Ireland) and were stored in digital format in two 12 channel dataloggers (MMS, Enschede, the Netherlands), using a sample frequency of $8 \mathrm{~Hz}$. At the end of the study all data were transferred to the hard disc of the computer.
Table 1 Radiographic, impedancometric, and $\mathrm{pH}$ metric data in controls and patients

\begin{tabular}{lll}
\hline & Controls & Patients \\
\hline Air bubble size AP fasting $\left(\mathrm{cm}^{2}\right)$ & $11.5(1.3)$ & $14.0(5.4)$ \\
Air bubble size L fasting $\left(\mathrm{cm}^{2}\right)$ & $9.9(3.5)$ & $18.3(9.1)$ \\
Air bubble size AP postprandial $\left(\mathrm{cm}^{2}\right)$ & $11.9(1.7)$ & $10.5(2.9)$ \\
Air bubble size L postprandial $\left(\mathrm{cm}^{2}\right)$ & $11.0(1.3)$ & $12.5(3.4)$ \\
Number of swallows fasting/h & $79.5(8.5)$ & $70.0(14.5)$ \\
Number of air swallows fasting/h & $21.5(3.4)$ & $30.4(9.4)$ \\
Number of liquid reflux fasting/h & $0.6(0.4)$ & $0.9(0.4)$ \\
Number of gas reflux fasting/h & $4.7(1.0)$ & $4.4(0.8)$ \\
Number of mixed reflux fasting/h & $0.8(0.3)$ & $3.4(2.8)$ \\
Number of swallows postprandial/h & $64.2(6.1)$ & $49.5(5.9)$ \\
Number of air swallows postprandial/h & $24.4(2.8)$ & $21.8(5.8)$ \\
Number of liquid reflux postprandial/h & $1.8(0.4)$ & $1.8(0.4)$ \\
Number of gas reflux postprandial/h & $3.0(0.6)$ & $3.7(0.8)$ \\
Number of mixed reflux postprandial/h $/ \mathrm{h}$ & $4.2(0.7)$ & $6.2(3.1)$ \\
Number of episodes with pH $<4$ & $5.6(1.2)$ & $3.0(1.4)$ \\
\% of time with $\mathrm{pH}<4$ & $6.0(2.3)$ & $4.3(2.0)$ \\
\hline
\end{tabular}

Radiographs were taken in anteroposterior (AP) and lateral (L) direction. Differences between controls and patients are not statistically significant.

\section{Data analysis}

The size of the gastric air bubble was measured from the anteroposterior and lateral radiographs using transparent millimeter blocked paper. The projection of the impedance catheter was used as a reference for actual size. In the impedance signals reflux episodes were identified and classified as liquid, gas, or mixed liquid-gas reflux based on previously described criteria. ${ }^{12}$ Furthermore, using the $\mathrm{pH}$ tracings, liquid reflux, and mixed liquid-gas reflux were classified as acidic or non-acidic, using a threshold of $\mathrm{pH}=4$. Percentage of time with $\mathrm{pH}<4$ was calculated using commercially available software (MMS, Enschede, the Netherlands). The period of meal consumption was not taken into account for analysis.

\section{Statistical analysis and presentation of data}

Pearson's test was used to calculate the degree of correlation between gastric air bubble size in anterior and lateral projection. Differences between healthy volunteers and patients were compared using the $t$ test and the MannWhitney test for parametric and non-parametric data respectively. The Wilcoxon test was used for comparisons between number of belches before and after the meal. Differences were considered statistically significant when $\mathrm{p} \leqslant 0.05$. Throughout the manuscript data are presented as mean (SEM)

\section{RESULTS}

Gastric air bubble surface areas measured radiographically in anteroposterior and in lateral projection correlated extremely well $(\mathrm{p}<0.001, r=0.978)$. There were no differences between gastric air bubble size in healthy volunteers and patients with excessive belching (table 1). Furthermore, there were no differences in the number of liquid and mixed reflux events between healthy controls and patients (table 1). Results of $\mathrm{pH}$ metric studies were also comparable between the two groups (table 1).

The rates of swallowing were similar in patients and controls, both before and after the meal (table 1). In approximately one third of the total number of swallows, a significant amount of air (impedance increase of $>1000 \Omega$ ) preceded the liquid bolus and moved from proximal to distal in the oesophagus (fig 2). The incidence of air swallowing was similar in healthy controls and in patients. Two types of retrograde air/gas flow were observed. The first type was characterised by a rapid increase in impedance that moved from distal to proximal (fig 3A), representing gas reflux from 


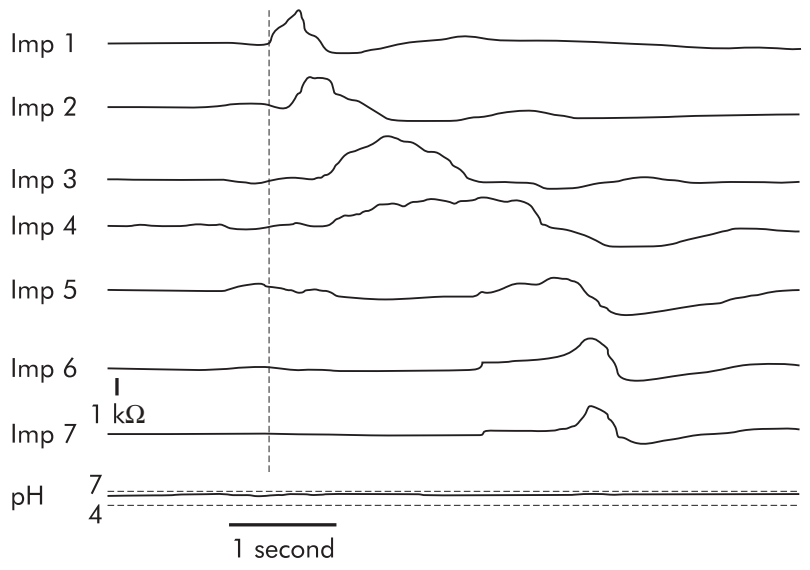

Figure 2 Air swallowing. Air (high impedance) is propulsed in front of a bolus (low impedance) and moves aborally.

the stomach. The frequency of this pattern was similar between patients and controls. The second type was characterised by a very rapid increase in impedance that moved from proximal to distal, followed by a retrograde decrease in impedance to baseline (fig 3B). This pattern was only observed in patients and not in controls and corresponded in time with the belches as indicated by the patients with the event marker. None of these belches was accompanied by impedancometric evidence of gastro-oesophageal reflux or venting of air from the stomach.

The second belch pattern differed from the pattern of air swallowing in that the increase in impedance was not moving at the velocity of a peristaltic wave but much faster $(1.37(0.19) \mathrm{m} / \mathrm{s})$ and in that the sudden increase of impedance moving in antegrade direction was followed by a decrease moving in retrograde direction. In $13.5 \%$ of cases the initial increase of impedance was followed by a peristaltic wave, obscuring the retrograde decrease. In 83.9 (2.3) \% of the supragastric belches the air reached the most distal impedance sensor. The average rate of belching was 40.9 (15.2) per hour before the meal and 67.7 (23.8) per hour after the meal, the difference not being statistically significant.
Manometry was performed concomitantly in four aerophagia patients. None of the supragastric belches was accompanied by a common cavity phenomenon or LOS relaxation. Instead, manometry revealed two distinct patterns. In $43.8 \%$ of the supragastric belches an initial decrease in oesophageal pressure preceded the increase in impedance. Invariably, this was followed by a pressure rise in all channels, including the intragastric channel (fig 4). In $42.0 \%$ of the supragastric belches a pharyngeal contraction could be recognised that preceded the increase in impedance and the simultaneous pressure increase in all channels (fig 5 ). After the pressure increase, when pressure reached baseline pressure, the rapid retrograde decrease in impedance occurred. In $10.9 \%$ of the supragastric belches both a pharyngeal contraction and a decrease in oesophageal pressure were observed. In the remaining few supragastric belches manometric tracings were not interpretable.

\section{DISCUSSION}

Excessive belching is often thought to result from aerophagia: swallowing of air too frequently or in too large quantities. In the Rome II consensus aerophagia is described as a repetitive pattern of swallowing or ingesting air and belching, usually an unconscious act unrelated to meals, and presumably a learned habit. ${ }^{14}$ Despite the fact that almost every gastroenterologist has seen patients with this disorder, surprisingly little research in this field has been performed and not much is known about the mechanisms involved. Generally, it is thought that excessive belching in these patients is the result of venting air from the stomach after a period of excessive air swallowing.

With multiple intraluminal impedance recordings we were able to demonstrate two different types of belching. The first type, characterised by an increment of intraluminal impedance moving in oral direction, represents venting of gas from the stomach and can be referred to as gastric belching. The second type, characterised by a rapid antegrade impedance rise followed by a rapid retrograde return to baseline, represents oesophageal air ingestion followed by immediate expulsion. As the ingested air does not reach the stomach in this type of belching, it can be referred to as supragastric belching. Whereas gastric belches were found both in patients and healthy volunteers, supragastric belches

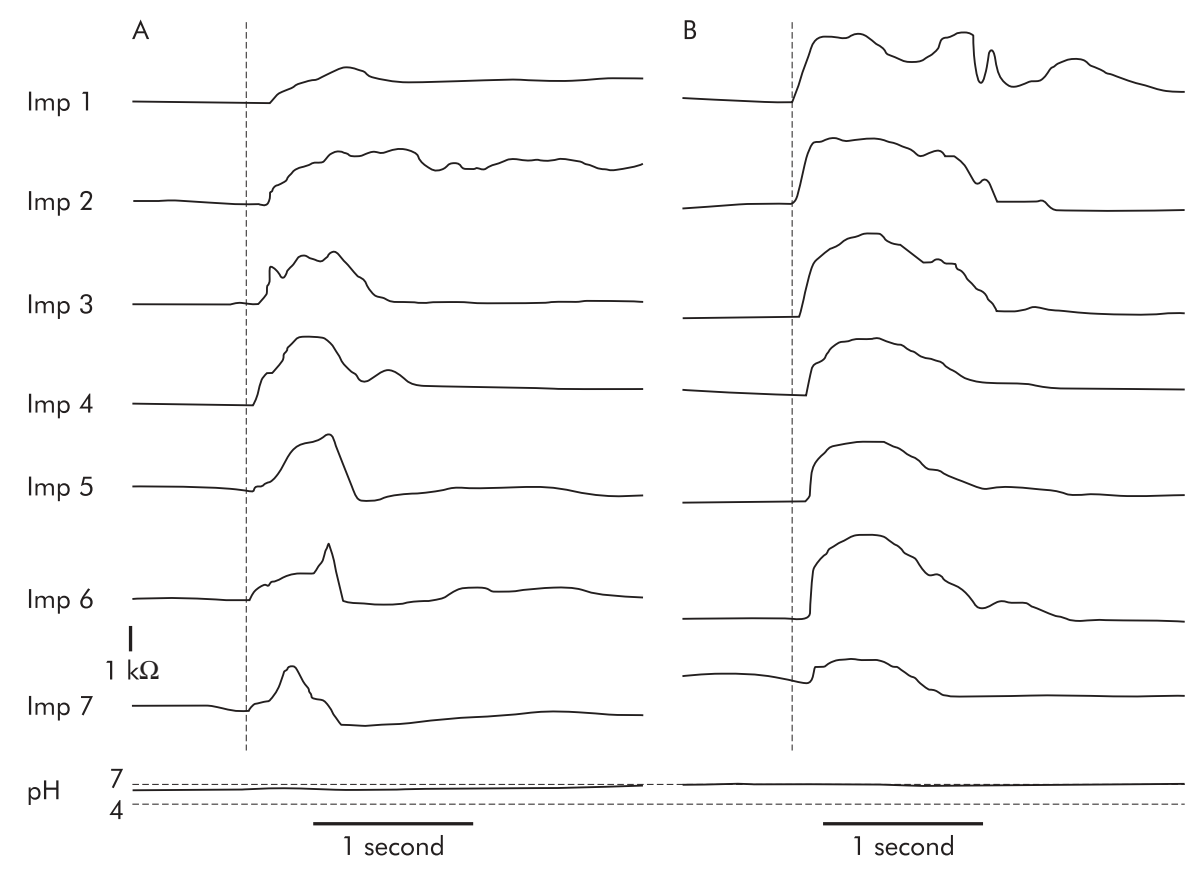

Figure 3 Two different types of belches. (A) The gastric belch is characterised by an increase in impedance that moves in oral direction. (B) A supragastric belch is characterised by an episode with high impedance, the onset of which starts in the proximal channels and moves distally; the end of which is cleared in the opposite direction. 


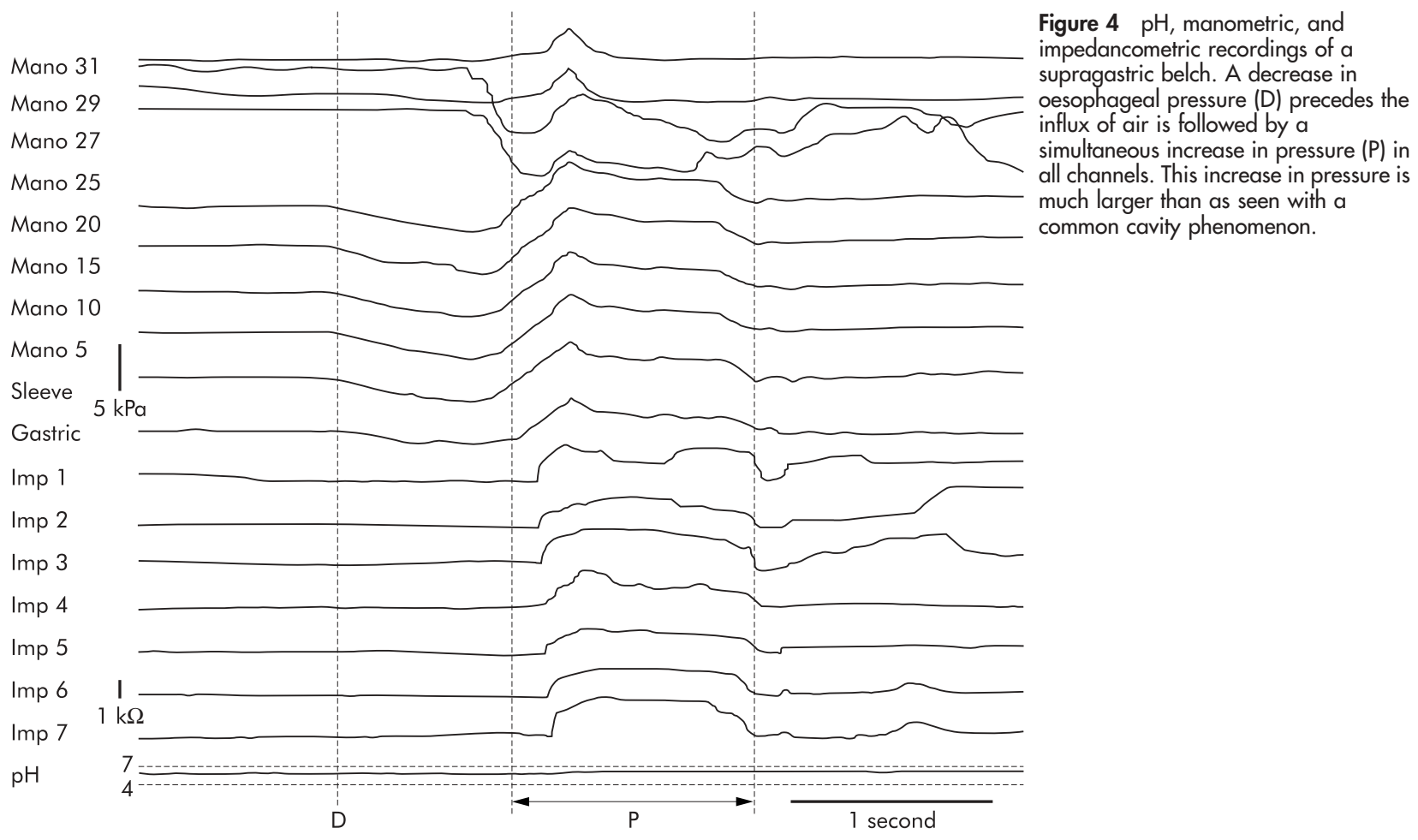

were only seen in patients with excessive belching (aerophagia). The observation that patients with excessive belching did not differ from healthy controls with respect to the swallowing of air and the size of the gastric air bubble on radiographic images supports the concept that the mechanism of belching in these patients does not involve the stomach. Likewise liquid and mixed reflux episodes were not more frequent in the patients with repetitive belching. Manometry did not show lower oesophageal sphincter relaxations or common cavity phenomenon at moments that patients pushed the event marker to indicate they had a belch.

The question remains what mechanism results in this air flow? This air could enter the oesophagus because of either a backward pushing force induced by a pharyngeal contraction $^{15}$ or by a forward sucking force generated by a negative pressure gradient in the oesophagus. Our manometric findings indicate that both mechanisms may play a role:

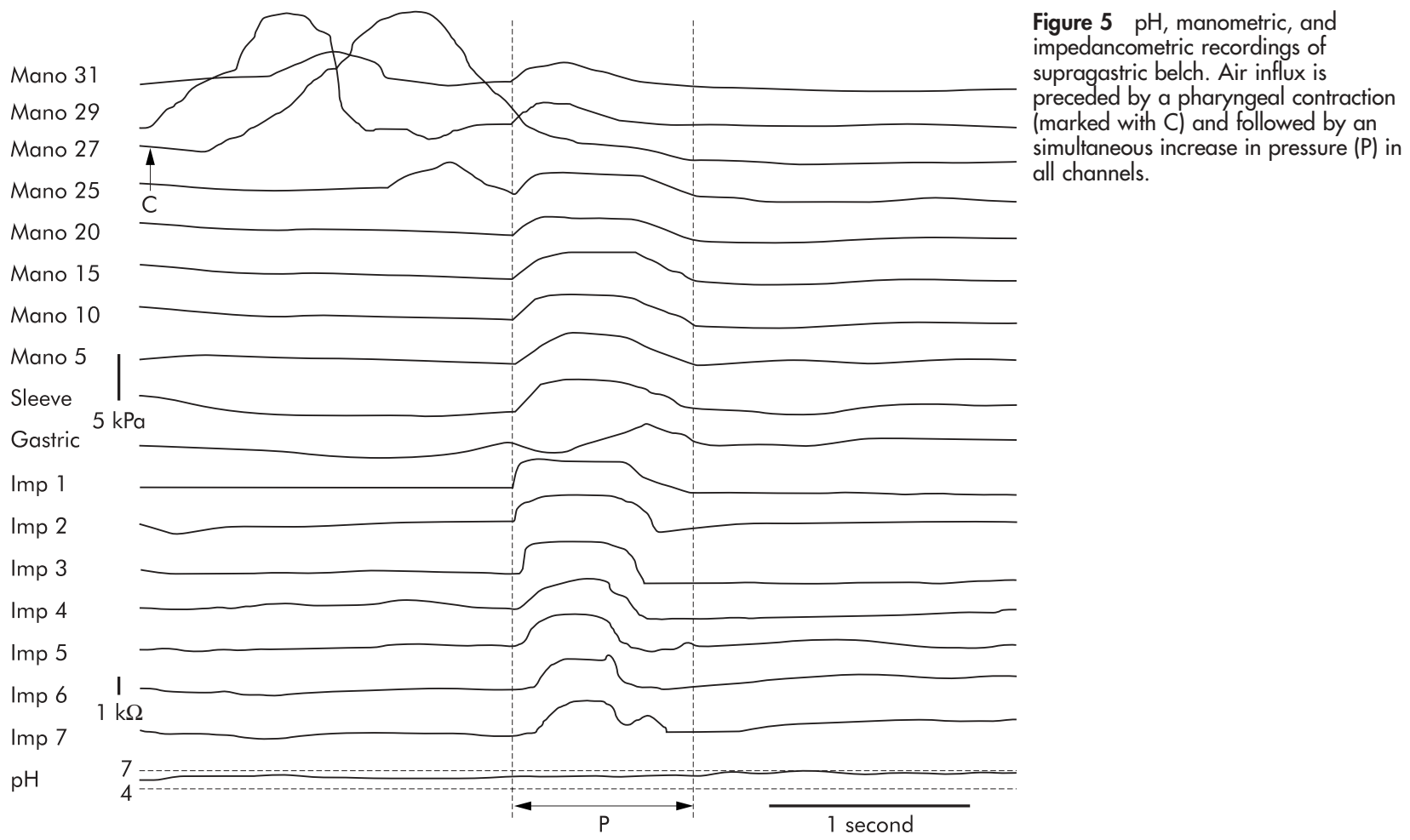


immediately before the increase in electrical impedance both pharyngeal contractions $(42.0 \%)$ and decreases in oesophageal body pressure $(43.8 \%)$, and combinations of the two mechanisms (10.9\%) were observed. In both mechanisms a sudden increase in abdominal and oesophageal pressure was observed that ended with the decrease in impedance. This pressure increase was simultaneously in the gastric and oesophageal channels and is presumably caused by an abdominal strain, necessary to evacuate the oesophageal air.

Most patients with excessive belching believe that their belching results from some kind of organic disorder. Often, patients are convinced that they have an unusually high production of gas in the stomach or intestine. Multiple intraluminal impedance monitoring makes it possible to obtain more insight in the mechanism of excessive belching in these patients. The incidence of supragastric belches did not increase significantly after the meal, in contrast to other functional disorders like functional dyspepsia or the rumination syndrome, where eating a meal triggers symptoms.

Complaints of excessive belching in patients with aerophagia and supragastric belching are probably not relieved by transient LOS relaxation inhibitors like baclofen, as these belches do not originate from the stomach. Instead, more is to be expected from treatment modalities such as behavioural therapy. ${ }^{16}$ Perhaps the incidence of supragastric belching can be reduced by making patients aware that these belches are self-induced. There might be a role for impedance monitoring in the development of a biofeedback treatment programme, because impedance monitoring makes it possible to differentiate between gastric and supragastric belches. Indeed, most patients are convinced their symptoms are caused by expulsion of gastric gas. Some physicians try to convince their patients of the fact that these belches are self-induced by showing them that they themselves can also belch. Anecdotally, it was reported that excessive belching was reduced with hypnosis as well. ${ }^{17}$ Some aerophagia patients report that, initially, belching relieved some kind of unpleasant sensation in the upper abdomen, but after a while the belching became bothersome itself. We suspect that excessive belching is some kind of learned behaviour and is initially induced consciously. After a while, however, this control is lost.

We can conclude that patients with excessive belching do not have more frequent gaseous reflux from stomach to oesophagus and pharynx than healthy subjects. Their repetitive and bothersome belches originate from a distinct belch pattern, characterised by air that does not reach the stomach.

\section{ACKNOWLEDGEMENTS}

The authors like to thank Ms A Baron for technical assistance. A J Bredenoord was supported by a clinical research trainee grant from Janssen-Cilag, the Netherlands.

\section{Authors' affiliations}

A J Bredenoord, B L A M Weusten, R Timmer, Department of Gastroenterology, Sint Antonius Hospital, Nieuwegein, the Netherlands D Sifrim, Center for Gastroenterology Research, Catholic University of Leuven, Leuven, Belgium

A J P M Smout, Gastrointestinal Research Unit, University Medical Center, Utrecht, the Netherlands

\section{REFERENCES}

1 Pouderoux P, Ergun GA, Lin S, et al. Esophageal bolus transit imaged by ultrafast computerized tomography. Gastroenterology 1996;110:1422-8.

2 Holloway RH, Hongo M, Berger K, et al. Gastric distention: a mechanism for postprandial gastroesophageal reflux. Gastroenterology 1985:89:779-84.

3 Wyman JB, Dent J, Heddle R, et al. Control of belching by the lower oesophageal sphincter. Gut 1990;31:639-46.

4 Straathof JW, Ringers J, Lamers CB, et al. Provocation of transient lower esophageal sphincter relaxations by gastric distension with air. Am J Gastroenterol 2001;96:2317-23.

5 McNally EF, Kelly E, Ingelfinger F. Mechanism of belching: effects of gastric distention with air. Gastroenterology 1964;46:254-9.

6 Scheffer RC, Akkermans LM, Bais JE, et al. Elicitation of transient lower oesophageal sphincter relaxations in response to gastric distension and meal ingestion. Neurogastroenterol Motil 2002; 14:647-55.

7 Kahrilas PJ, Dodds WJ, Dent J, et al. Upper esophageal sphincter function during belching. Gastroenterology 1986;91:133-40.

8 Tew S, Ackroyd R, Jamieson GG, et al. Belching and bloating: facts and fantasy after antireflux surgery. Br J Surg 2000;87:477-81.

9 Anonymous. Physiology of belch. Lancet 1991;337:23-4.

10 Enzmann DR, Harell GS, Zboralske FF. Upper esophageal responses to intraluminal distention in man. Gastroenterology 1977;72:1292-8.

11 Lin M, Triadafilopoulos G. Belching: dyspepsia or gastroesophageal reflux disease? Am J Gastroenterol 2003:98:2139-45.

12 Fass J, Silny J, Braun J, et al. Measuring esophageal motility with a new intraluminal impedance device. First clinical results in reflux patients. Scand J Gastroenterol 1994;29:693-702.

13 Sifrim D, Silny J, Holloway RH, et al. Patterns of gas and liquid reflux during transient lower oesophageal sphincter relaxation: a study using intraluminal electrical impedance. Gut 1999;44:47-54.

14 Talley NJ, Stanghellini V, Heading RC, et al. Functional gastroduodenal disorders. Gut 1999:45(Suppl 2):1137-42.

15 Fisher MA, Hendrix TR, Hunt JN, et al. Relation between volume swallowed and velocity of the bolus ejected from the pharynx into the esophagus. Gastroenterology 1978;74:1238-40

16 Bassotti G, Whitehead WE. Biofeedback as a treatment approach to gastrointestinal tract disorders. Am J Gastroenterol 1994;89:158-64

17 Spiegel SB. Uses of hypnosis in the treatment of uncontrollable belching: a case report. Am J Clin Hypn 1996;38:263-70.

\section{EDITOR'S QUIZ: GI SNAPSHOT}

\section{Answer}

From question on page 1552

At endoscopy, rectal hamartomatous polyps appeared as multiple pink or translucent sessile polyps, measuring less than $5 \mathrm{~mm}$ (fig 1A). On microscopic examination (fig 1B), the hamartomatous polyps consisted of hyperplastic dilated glands lined by mucin secreting cylindrical cells, lying in oedematous lamina propria, with smooth muscle fibres arborising between the crypts. A diagnosis of rectal hamartomatous polyposis in a patient with tuberous sclerosis complex (TSC) was made.

TSC is a rare dominantly inherited disorder characterised by the presence of hamartomas in multiple organs. Involvement of the gastrointestinal tract is unusual. Rectal hamartomatous polyposis has rarely been reported in the literature but its prevalence is probably underestimated. The polyps are asymptomatic and usually fortuitously discovered at endoscopy. They can sometimes be palpable during digital rectal examination. Rectal hamartomatous polyposis has no malignant potential. In the latest revised diagnostic criteria for TSC, it has been categorised in the minor features for diagnosis of this disease. 